\title{
Analysis of two-dimensional photonic crystals using a multidomain pseudospectral method
}

\author{
Po-jui Chiang, ${ }^{1}$ Chin-ping Yu, ${ }^{1}$ and Hung-chun Chang ${ }^{1,2,3, *}$ \\ ${ }^{1}$ Graduate Institute of Electro-Optical Engineering, National Taiwan University, Taipei, Taiwan 106-17, Republic of China \\ ${ }^{2}$ Graduate Institute of Communication Engineering, National Taiwan University, Taipei, Taiwan 106-17, Republic of China \\ ${ }^{3}$ Department of Electrical Engineering, National Taiwan University, Taipei, Taiwan 106-17, Republic of China
}

(Received 18 April 2006; revised manuscript received 21 November 2006; published 20 February 2007)

\begin{abstract}
An analysis method based on a multidomain pseudospectral method is proposed for calculating the band diagrams of two-dimensional photonic crystals and is shown to possess excellent numerical convergence behavior and accuracy. The proposed scheme utilizes the multidomain Chebyshev collocation method. By applying Chebyshev-Lagrange interpolating polynomials to the approximation of spatial derivatives at collocation points, the Helmholtz equation is converted into a matrix eigenvalue equation which is then solved for the eigenfrequencies by the shift inverse power method. Suitable multidomain division of the computational domain is performed to deal with general curved interfaces of the permittivity profile, and field continuity conditions are carefully imposed across the dielectric interfaces. The proposed method shows uniformly excellent convergence characteristics for both the transverse-electric and transverse-magnetic waves in the analysis of different structures. The analysis of a mini band gap is also shown to demonstrate the extremely high accuracy of the proposed method.
\end{abstract}

DOI: 10.1103/PhysRevE.75.026703

PACS number(s): 02.70.Hm, 03.50.De

\section{INTRODUCTION}

Band structures are essential characteristics of photonic crystals (PCs), from which possible photonic band gaps (PBGs) can be identified [1-3]. For frequencies within the PBGs, wave propagation is forbidden and many photonic devices have been proposed and designed based on this phenomenon. In particular, two-dimensional (2D) PCs composed of either dielectric rods or air columns have been widely employed in many applications such as waveguiding, resonant cavity formation, and wavelength filtering. In this paper, we propose an analysis scheme with excellent numerical convergence behavior and accuracy for calculating the band structures of 2D PCs. The currently most used numerical methods for such calculations have been the plane-wave expansion (PWE) method [3-6] and the finite-difference time-domain (FDTD) method $[7,8]$. The finite-difference eigenvalue problem formulation has also been employed by Yang [9] and Shen et al. [10], and more recently by Yu and Chang [11] based on the Yee mesh as often employed in the FDTD method [12]. The Yee-mesh-based formulation was named the finite-difference frequency-domain (FDFD) method. Yu and Chang [11] used the FDFD method to analyze the band structures of 2D PCs with either square or triangular lattices and adopted a fourth-order accurate compact finite-difference scheme [13] to increase numerical efficiency and accuracy. Although the FDFD method offers results with accuracy comparable to those obtained using the MIT photonic-bands (MPB) package [14] based on the PWE method, the numerical convergent speed was found not to be uniformly fast among different bands in the two methods.

The numerical formulation proposed in this paper is based on the multidomain pseudospectral method using Chebyshev polynomials. The pseudospectral method has recently at-

*Electronic address: hcchang@cc.ee.ntu.edu.tw tracted raised attention as an alternative treatment for computational electromagnetics because of its high-order accuracy and fast convergence behavior over traditional techniques while retaining formulation simplicity. It has a long history of being applied to fluid dynamics [15] and has recently been extended to the analysis of electromagnetics both in the time [16-18] and in the frequency domain [19]. However, while the theory of the pseudospectral method has been well elaborated, the application in the frequency domain has not received much focus compared with that in the time domain in the electromagnetics community. In [19], the pseudospectral frequency-domain method was proposed and applied to solve the nonhomogeneous (nonzero-source) Helmholtz equation in a simple two-subdomain problem with rectangular structure shape. Our proposed scheme in this paper utilizes the multidomain Chebyshev collocation method supported by the curvilinear mapping technique [20] to facilitate and ameliorate the simulation of 2D PCs of arbitrary permittivity profile. The formulation is derived in the form of an eigenvalue problem so that we can readily obtain the eigenmodes by available mathematical tools. Here, we adopt the shift inverse power method (SIPM) for its particularly fast convergence characteristic over other conventional methods applying matrix inversion. Both the multidomain pseudospectral algorithm and the SIPM furnish our pseudospectral mode solver (PSMS) as a quite powerful and flexible method. To obtain high-accuracy full-vectorial modal solutions for dielectric structures, proper satisfaction of dielectric interface conditions is essential, whether it is based on the finite-difference method [21,22], the finite-element method [23], or others. Such proper treatment of interface conditions will be carefully considered in our formulation.

The rest of this paper is outlined as follows. The physical problem involving the Helmholtz equations is described in Sec. II along with the required Dirichlet and Neumann type boundary conditions across the dielectric interfaces. The formulation of the PSMS is presented in Sec. III. Numerical examples including 2D PCs with either a square or a trian- 


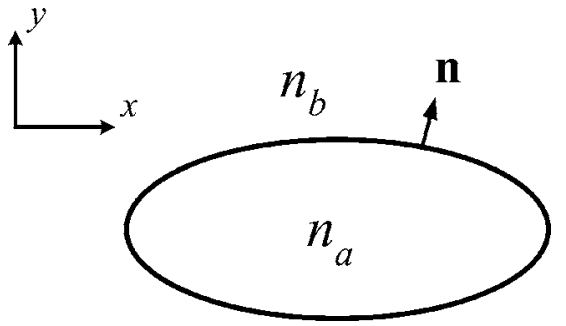

FIG. 1. Boundary between two homogeneous regions with refractive indices $n_{a}$ and $n_{b}$ within the unit cell. $\mathbf{n}$ is a unit vector normal to the boundary.

gular lattice and a high-accuracy analysis of a mini band gap are given and compared with other numerical methods in Sec. IV. The conclusion is drawn in Sec. V.

\section{THE PHYSICAL EQUATIONS}

Assume that the 2D PC considered is composed of lossless isotropic dielectric materials, and is uniform along the $z$ direction and periodic in the transverse $x-y$ plane. We will only consider the in-plane propagation that has zero propagation constant in the $z$ direction so that the wave modes in the PC are either transverse electric (TE) or transverse magnetic (TM) to the $z$ modes. To obtain the band structures, we need only solve the electromagnetic problem within a $2 \mathrm{D}$ unit cell. Assume that the PC possesses a piecewise uniform refractive index distribution $n$; Fig. 1 shows a boundary of arbitrary shape between two homogeneous regions within the unit cell with $n=n_{a}$ and $n=n_{b}$, respectively, and the same permeability as in free space. For TM modes, only $E_{z}, H_{x}$, and $H_{y}$ components exist. For TE modes, only $H_{z}, E_{x}$, and $E_{y}$ components exist. The fields are governed by the Helmholtz equation

$$
\left(\nabla^{2} / n^{2}\right) \phi(x, y)=-k_{0}^{2} \phi(x, y)
$$

where $\boldsymbol{\nabla}$ is the del operator, $k_{0}=\omega / c$ is the free-space wave number, $\omega$ is the angular frequency, $c$ is the speed of light in vacuum, and $\phi=H_{z}$ and $E_{z}$ for the TE and TM modes, respectively. By the Chebyshev collocation method which will be introduced in Sec. III, Eq. (1) leads to an $\omega / c$-formulation matrix eigenvalue equation of the form

$$
[P]\{\phi\}=-(\omega / c)^{2}\{\phi\}
$$

where $\{\phi\}$ is a vector composed of $\phi$ values at grid points and $[P]$ is the operator matrix. The eigenvalues can be solved by applying the SIPM.

We now discuss the boundary conditions for the TE and TM modes. Since the tangential fields $\left(H_{z}\right.$ or $\left.E_{z}\right)$ should be made continuous across the dielectric interfaces, referring to regions $a$ and $b$ in Fig. 1, we have the Dirichlet type boundary conditions

$$
H_{z}^{a}=H_{z}^{b}
$$

for the TE mode and

$$
E_{z}^{a}=E_{z}^{b}
$$

for the TM mode. We have another continuity condition in terms of the transverse components

$$
\mathbf{n} \times\left(E_{x}^{a} \hat{x}+E_{y}^{a} \hat{y}\right)=\mathbf{n} \times\left(E_{x}^{b} \hat{x}+E_{y}^{b} \hat{y}\right)
$$

for the TE mode and

$$
\mathbf{n} \times\left(H_{x}^{a} \hat{x}+H_{y}^{a} \hat{y}\right)=\mathbf{n} \times\left(H_{x}^{b} \hat{x}+H_{y}^{b} \hat{y}\right)
$$

for the TM mode, where $\mathbf{n}=n_{x} \hat{x}+n_{y} \hat{y}$ is the normal unit vector to the dielectric interface. From Maxwell's curl equations, we have

$$
\begin{gathered}
j \omega n^{2} E_{x}=\frac{\partial H_{z}}{\partial y}, \\
j \omega n^{2} E_{y}=-\frac{\partial H_{z}}{\partial x}
\end{gathered}
$$

for the TE mode and

$$
\begin{gathered}
j \omega \mu_{0} H_{x}=-\frac{\partial E_{z}}{\partial y}, \\
j \omega \mu_{0} H_{y}=\frac{\partial E_{z}}{\partial x}
\end{gathered}
$$

for the TM mode. By substituting Eqs. (5a)-(5d) into Eqs. (4a) and (4b), we derive the following Neumann type boundary conditions

$$
n_{x} \frac{\partial H_{z}^{a}}{\partial x}+n_{y} \frac{\partial H_{z}^{a}}{\partial y}=\left(\frac{n_{a}}{n_{b}}\right)^{2}\left(n_{x} \frac{\partial H_{z}^{b}}{\partial x}+n_{y} \frac{\partial H_{z}^{b}}{\partial y}\right)
$$

for the TE mode and

$$
n_{x} \frac{\partial E_{z}^{a}}{\partial x}+n_{y} \frac{\partial E_{z}^{a}}{\partial y}=\left(n_{x} \frac{\partial E_{z}^{b}}{\partial x}+n_{y} \frac{\partial E_{z}^{b}}{\partial y}\right)
$$

for the TM mode.

Due to the periodic geometry, we need to consider periodic boundary conditions (PBCs) at the boundaries of the unit cell for the field distribution in the PC. We will describe the PBCs for the respective cases in Sec. IV. In our numerical scheme, we combine the adjacent regions by imposing the Dirichlet and Neumann type boundary conditions on two sides of the interface to guarantee numerical stability.

\section{THE MULTIDOMAIN PSEUDOSPECTRAL METHOD}

The construction of the multidomain spectral collocation scheme for solving the Helmholtz equation is described in this section. The procedure to obtain a general form of the $[P]$ matrix in such a multidomain scheme will be discussed in detail.

\section{A. The Chebyshev spectral method}

For achieving better approximation characteristics in solving partitial differential equations, we select the widely used 
Chebyshev collocation method described in [15] as the basis of our numerical scheme. The Chebyshev polynomial $T_{N}(x)$ of degree $N$ is defined as

$$
T_{N}(x)=\cos \left(N \cos ^{-1} x\right)
$$

where $|x| \leqslant 1$, and the collocation points are given by the Chebyshev-Gauss-Lobatto points, defined as the roots of the polynomial $\left(1-x^{2}\right) T_{N}^{\prime}(x)$, where the prime means the derivative. One of the merits of employing Chebyshev polynomials is the existence of the analytical formula for their collocation points, given by

$$
x_{i}=\cos \left(\frac{i \pi}{N}\right), \quad i=0,1,2, \ldots, N .
$$

The Chebyshev collocation method provides a means to approximate the function $f(x)$ by global Chebyshev-Lagrange interpolating polynomials of degree $N$,

$$
f(x) \approx \sum_{i=0}^{N} f\left(x_{i}\right) g_{i}(x),
$$

where the interpolating Chebyshev-Lagrange polynomials are given by

$$
g_{i}(x)=\frac{\left(1-x^{2}\right) T_{N}^{\prime}(x)(-1)^{i+1}}{c_{i} N^{2}\left(x-x_{i}\right)}
$$

with $c_{0}=c_{N}=2$ and $c_{i}=1$ for $1 \leqslant i \leqslant N-1$. Then, with the interpolation in Eq. (10), the spatial derivatives of $f(x)$ at a collocation point $x_{i}$ can be computed by a matrix operator with element entries $D_{i j}=g_{j}^{\prime}\left(x_{i}\right)$, i.e.,

$$
\frac{d f\left(x_{i}\right)}{d x} \approx \sum_{j=0}^{N} g_{j}^{\prime}\left(x_{i}\right) f\left(x_{j}\right)=\sum_{j=0}^{N} D_{i j} f\left(x_{j}\right)
$$

where the explicit expression for $D_{i j}$ is given in [15] as

$$
D_{i j}=\left\{\begin{array}{lc}
\frac{c_{i}}{c_{j}} \frac{(-1)^{i+j}}{x_{i}-x_{j}}, & i \neq j, \\
\frac{-x_{i}}{2\left(1-x_{i}^{2}\right)}, & 1 \leqslant i=j \leqslant N-1, \\
\frac{2 N^{2}+1}{6}, & i=j=0, \\
-\frac{2 N^{2}+1}{6}, & i=j=N .
\end{array}\right.
$$

To extend the one-dimensional (1D) formula to a higherdimensional problem, matrix products should be the most convenient treatment for computational simplicity. For the 2D PC analysis, we need to consider a 2D setting and define the approximation to $f(x, y)$ as

$$
f(x, y) \approx \sum_{i=0}^{M} \sum_{j=0}^{N} f\left(x_{i}, y_{j}\right) g_{i}(x) g_{j}(y)
$$

where the Chebyshev-Gauss-Lobatto grid $y_{i}$ has been introduced. This approach has the benefit that the derivatives can be calculated through the 1D formula Eq. (11) and thus the differential formulas at the 2D collocation points arranged in a rectangular domain can be expressed as in the following matrix multiplication form:

$$
\begin{aligned}
& {\left[\begin{array}{cccc}
\frac{\partial f\left(x_{0}, y_{0}\right)}{\partial x} & \frac{\partial f\left(x_{0}, y_{1}\right)}{\partial x} & \cdots & \frac{\partial f\left(x_{0}, y_{N}\right)}{\partial x} \\
\frac{\partial f\left(x_{1}, y_{0}\right)}{\partial x} & \frac{\partial f\left(x_{1}, y_{1}\right)}{\partial x} & \cdots & \frac{\partial f\left(x_{1}, y_{N}\right)}{\partial x} \\
\vdots & \vdots & \cdots & \vdots \\
\frac{\partial f\left(x_{M}, y_{0}\right)}{\partial x} & \frac{\partial f\left(x_{M}, y_{1}\right)}{\partial x} & \cdots & \frac{\partial f\left(x_{M}, y_{N}\right)}{\partial x}
\end{array}\right]=\frac{\partial \overline{\bar{f}}_{\text {rect }}}{\partial x}} \\
& =\overline{\bar{D}}_{(M+1) \times(M+1)} \\
& {\left[\begin{array}{cccc}
\frac{\partial f\left(x_{0}, y_{0}\right)}{\partial y} & \frac{\partial f\left(x_{0}, y_{1}\right)}{\partial y} & \cdots & \frac{\partial f\left(x_{0}, y_{N}\right)}{\partial y} \\
\frac{\partial f\left(x_{1}, y_{0}\right)}{\partial y} & \frac{\partial f\left(x_{1}, y_{1}\right)}{\partial y} & \cdots & \frac{\partial f\left(x_{1}, y_{N}\right)}{\partial y} \\
\vdots & \vdots & \cdots & \vdots \\
\frac{\partial f\left(x_{M}, y_{0}\right)}{\partial y} & \frac{\partial f\left(x_{M}, y_{1}\right)}{\partial y} & \cdots & \frac{\partial f\left(x_{M}, y_{N}\right)}{\partial y}
\end{array}\right]=\frac{\partial \overline{\bar{f}}_{\text {rect }}}{\partial y}} \\
& =\overline{\bar{f}}_{\text {rect }} \overline{\bar{D}}_{(N+1) \times(N+1)}^{T}, \\
&
\end{aligned}
$$

where $\overline{\bar{f}}_{\text {rect }}$ is an $(M+1) \times(N+1)$ matrix with entries $f\left(x_{i}, y_{j}\right)$, $i=0,1,2, \ldots, M$ and $j=0,1,2, \ldots, N$, corresponding to collocation points in rectangular arrangement, $\overline{\bar{D}}_{(M+1) \times(M+1)}$ is an $(M+1) \times(M+1)$ matrix with entries $D_{i j}$ as defined by Eq. (12) but with $N$ replaced by $M$, and the superscript $T$ denotes the transpose. However, the employment of matrix products is still restricted by the nature of rectangular grids, which is unsound to deal with curved boundaries. In the next section, a curvilinear representation will be introduced to overcome this barrier.

\section{B. The curvilinear representation}

In order to extend our numerical scheme defined above on rectangular grids to the analysis of structures with curved boundaries, a multidomain formulation and modified differential matrices will be established. We first split the whole computational domain into a series of nonoverlapping curvilinear quadrilaterals according to the profile and material distributions. Then by applying the transfinite blending function presented in [20], each curvilinear quadrilateral in Cartesian $(x, y)$ coordinates can be mapped onto a unit square one $[-1,1] \times[-1,1]$ in curvilinear $(\xi, \eta)$ coordinates, as shown in Fig. 2, under the transformation

$$
\xi=\xi(x, y), \quad \eta=\eta(x, y) .
$$

It yields the great advantage that we can thus calculate the spatial derivatives $\partial f(x, y) / \partial x$ and $\partial f(x, y) / \partial y$ in a subdomain with curved boundaries by the formulation defined on this unit element. By the fundamental differential principle, the modified differential matrices are expressed as 


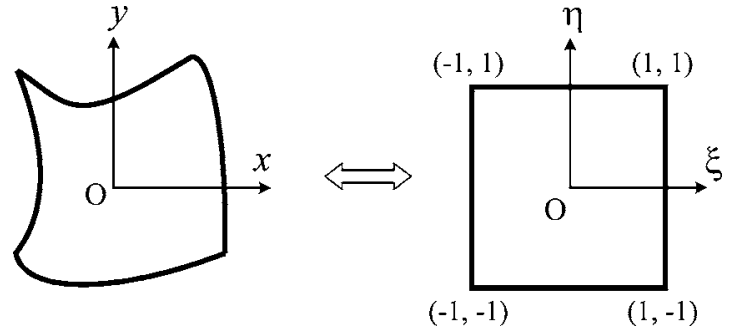

FIG. 2. Illustration of the domain mapping between a curvilinear quadrilateral in the Cartesian coordinates $(x, y)$ and a unit square one $[-1,1] \times[-1,1]$ in the general curvilinear coordinates $(\xi, \eta)$.

$$
\begin{aligned}
\frac{\partial \xi(x, y)}{\partial x} \frac{\partial}{\partial \xi} & \approx \overline{\bar{D}}_{\xi x} \\
& =\left[\begin{array}{cccc}
\xi_{00}^{x} D_{00} & \xi_{01}^{x} D_{01} & \cdots & \xi_{0 M}^{x} D_{0 M} \\
\xi_{10}^{x} D_{10} & \xi_{11}^{x} D_{11} & \cdots & \xi_{1 M}^{x} D_{1 M} \\
\vdots & \vdots & \cdots & \vdots \\
\xi_{M 0}^{x} D_{M 0} & \xi_{M 1}^{x} D_{M 1} & \cdots & \xi_{M M}^{x} D_{M M}
\end{array}\right]_{(M+1) \times(M+1)},
\end{aligned}
$$

$$
\begin{aligned}
\frac{\partial \eta(x, y)}{\partial x} \frac{\partial}{\partial \eta} & \approx \overline{\bar{D}}_{\eta x} \\
& =\left[\begin{array}{cccc}
\eta_{00}^{x} D_{00} & \eta_{01}^{x} D_{10} & \cdots & \eta_{0 N}^{x} D_{N 0} \\
\eta_{10}^{x} D_{01} & \eta_{11}^{x} D_{11} & \cdots & \eta_{1 N}^{x} D_{N 1} \\
\vdots & \vdots & \cdots & \vdots \\
\eta_{N 0}^{x} D_{0 N} & \eta_{N 1}^{x} D_{1 N} & \cdots & \eta_{N N}^{x} D_{N N}
\end{array}\right]_{(N+1) \times(N+1)},
\end{aligned}
$$

$$
\begin{aligned}
\frac{\partial \xi(x, y)}{\partial y} \frac{\partial}{\partial \xi} & \approx \overline{\bar{D}}_{\xi y} \\
& =\left[\begin{array}{cccc}
\xi_{00}^{y} D_{00} & \xi_{01}^{y} D_{01} & \cdots & \xi_{0 M}^{y} D_{0 M} \\
\xi_{10}^{y} D_{10} & \xi_{11}^{y} D_{11} & \cdots & \xi_{1 M}^{y} D_{1 M} \\
\vdots & \vdots & \cdots & \vdots \\
\xi_{M 0}^{y} D_{M 0} & \xi_{M 1}^{y} D_{M 1} & \cdots & \xi_{M M}^{y} D_{M M}
\end{array}\right]_{(M+1) \times(M+1)},
\end{aligned}
$$

$$
\begin{aligned}
\frac{\partial \eta(x, y)}{\partial y} \frac{\partial}{\partial \eta} & \approx \overline{\bar{D}}_{\eta y} \\
& =\left[\begin{array}{cccc}
\eta_{00}^{y} D_{00} & \eta_{01}^{y} D_{10} & \cdots & \eta_{0 N}^{y} D_{N 0} \\
\eta_{10}^{y} D_{01} & \eta_{11}^{y} D_{11} & \cdots & \eta_{1 N}^{y} D_{N 1} \\
\vdots & \vdots & \cdots & \vdots \\
\eta_{N 0}^{y} D_{0 N} & \eta_{N 1}^{y} D_{1 N} & \cdots & \eta_{N N}^{y} D_{N N}
\end{array}\right]_{(N+1) \times(N+1)},
\end{aligned}
$$

where

$$
\xi_{i j}^{x}=\partial \xi\left(x_{i}, y_{j}\right) / \partial x, \quad \eta_{i j}^{x}=\partial \eta\left(x_{i}, y_{j}\right) / \partial x,
$$

$$
\xi_{i j}^{y}=\partial \xi\left(x_{i}, y_{j}\right) / \partial y, \quad \eta_{i j}^{y}=\partial \eta\left(x_{i}, y_{j}\right) / \partial y,
$$

and $D_{i j}$ is the matrix element previously defined in Eq. (12). We can thus obtain the approximations for the derivatives of $f(x, y)$ in a single subdomain by the operators defined in the $(\xi, \eta)$ coordinates as

$$
\begin{aligned}
& \frac{\partial \overline{\bar{f}}_{\text {cur }}}{\partial x}=\overline{\bar{D}}_{x} \overline{\bar{f}}_{\text {cur }}=\overline{\bar{D}}_{\xi x} \overline{\bar{f}}_{\text {cur }}+\overline{\bar{f}}_{\text {cur }} \overline{\bar{D}}_{\eta x}, \\
& \frac{\partial \overline{\bar{f}}_{\text {cur }}}{\partial y}=\overline{\bar{D}}_{y} \overline{\bar{f}}_{\text {cur }}=\overline{\bar{D}}_{\xi y} \overline{\bar{f}}_{\text {cur }}+\overline{\bar{f}}_{\text {cur }} \overline{\bar{D}}_{\eta y} .
\end{aligned}
$$

Here the matrix $\overline{\bar{f}}_{\text {cur }}$ is in general different from $\overline{\bar{f}}_{\text {rect }}$ in Eq. (14) in that the latter has been defined on rectangular grids while the former is on deformed locations of grids adapted to the shape of the subdomain, and thus the operator matrices $\overline{\bar{D}}_{x}$ and $\overline{\bar{D}}_{y}$ are different from the $\overline{\bar{D}}$ matrices in Eq. (14). We finally have the discrete form of Eq. (1) in a single subdomain via Eq. (17) as

$$
n^{-2}\left[\overline{\bar{D}}_{x}\left(\overline{\bar{D}}_{x} \overline{\bar{\Phi}}\right)+\overline{\bar{D}}_{y}(\overline{\bar{D}} \overline{\bar{\Phi}})\right]=-(\omega / c)^{2} \overline{\bar{\Phi}}
$$

where the matrix $\overline{\bar{\Phi}}$ has entries that are the values of the $2 \mathrm{D}$ field distribution $\phi(x, y)$ at deformed locations of grids.

\section{Formulation of the PSMS}

Equation (18) is not in the form of an eigenvalue problem and thus cannot be easily solved numerically. In the following, we shall relocate the matrix elements (while retaining the same values) to convert Eq. (18) into a resolvable form of Eq. (2). We shall start with a single-domain setting and then extend to a multidomain environment.

First, we rearrange the $(M+1) \times(N+1)$ matrix $\overline{\bar{\Phi}}$ in Eq. (18) into the $[(M+1)(N+1)]$ column vector $\{\phi\}$ as in Eq. (2) as below:

$$
\begin{aligned}
\overline{\bar{\Phi}} & =\left[\begin{array}{cccc}
\Phi_{00} & \Phi_{01} & \cdots & \Phi_{0 N} \\
\Phi_{10} & \Phi_{11} & \cdots & \Phi_{1 N} \\
\vdots & \vdots & \cdots & \vdots \\
\Phi_{M 0} & \Phi_{M 1} & \cdots & \Phi_{M N}
\end{array}\right]_{(M+1) \times(N+1)} \Rightarrow\{\phi\} \\
& =\left[\begin{array}{c}
\Phi_{00} \\
\Phi_{10} \\
\vdots \\
\Phi_{M 0} \\
\Phi_{01} \\
\vdots \\
\Phi_{M N}
\end{array}\right]_{[(M+1)(N+1)] \times 1}
\end{aligned}
$$

The operator matrix $[P]$ in Eq. (2) can be expressed in terms of other operators in Eq. (18) with slight modification 


$$
[P]=n^{-2}\left[\overline{\bar{D}}_{x 00} \overline{\bar{D}}_{x 00}+\overline{\bar{D}}_{y 00} \overline{\bar{D}}_{y 00}\right]_{k \times k} .
$$

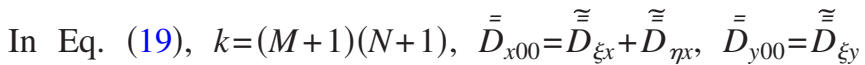
$+\stackrel{\bar{D}}{D}_{\eta y}$,

$$
\begin{aligned}
& \stackrel{\bar{D}}{\xi x}_{\xi x}=\left[\begin{array}{ccc}
\overline{\bar{D}}_{\xi x} & \mathbf{0} & \mathbf{0} \\
\mathbf{0} & \ddots & \mathbf{0} \\
\mathbf{0} & \mathbf{0} & \overline{\bar{D}}_{\xi x}
\end{array}\right]_{k \times k}, \\
& \stackrel{\overline{\bar{D}}}{\xi y}_{\bar{\xi}}=\left[\begin{array}{ccc}
\overline{\bar{D}}_{\xi y} & \mathbf{0} & \mathbf{0} \\
\mathbf{0} & \ddots & \mathbf{0} \\
\mathbf{0} & \mathbf{0} & \overline{\bar{D}}_{\xi y}
\end{array}\right]_{k \times k} \text {, } \\
& \stackrel{\bar{D}}{\eta x}_{\eta x}=\left[\begin{array}{cccc}
\operatorname{tr}\left[D_{\eta x(0,0)}\right] & \operatorname{tr}\left[D_{\eta x(0,1)}\right] & \ldots & \operatorname{tr}\left[D_{\eta x(0, N)}\right] \\
\operatorname{tr}\left[D_{\eta x(1,0)}\right] & \operatorname{tr}\left[D_{\eta x(1,1)}\right] & \ldots & \operatorname{tr}\left[D_{\eta x(1, N)}\right] \\
\vdots & \vdots & \vdots & \vdots \\
\operatorname{tr}\left[D_{\eta x(N, 0)}\right] & \operatorname{tr}\left[D_{\eta x(N, 1)}\right] & \ldots & \operatorname{tr}\left[D_{\eta x(N, N)}\right]
\end{array}\right]_{k \times k} \\
& \widetilde{\bar{D}}_{\eta y}=\left[\begin{array}{cccc}
\operatorname{tr}\left[D_{\eta y(0,0)}\right] & \operatorname{tr}\left[D_{\eta y(0,1)}\right] & \ldots & \operatorname{tr}\left[D_{\eta y(0, N)}\right] \\
\operatorname{tr}\left[D_{\eta y(1,0)}\right] & \operatorname{tr}\left[D_{\eta y(1,1)}\right] & \ldots & \operatorname{tr}\left[D_{\eta y(1, N)}\right] \\
\vdots & \vdots & \vdots & \vdots \\
\operatorname{tr}\left[D_{\eta y(N, 0)}\right] & \operatorname{tr}\left[D_{\eta y(N, 1)}\right] & \ldots & \operatorname{tr}\left[D_{\eta y(N, N)}\right]
\end{array}\right]_{k \times k},
\end{aligned}
$$

where $\quad \overline{\bar{D}}_{\xi x}, \quad \overline{\bar{D}}_{\xi y}, \quad D_{\eta x(i, j)}=\eta_{i j}^{x} D_{i j}(i=1,2, \ldots, N, j$ $=1,2, \ldots, N), \quad$ and $\quad D_{\eta y(i, j)}=\eta_{i j}^{y} D_{i j}(i=1,2, \ldots, N, j$ $=1,2, \ldots, N)$ have been given in Eq. (16), and $\operatorname{tr}[a]$ is a diagonal matrix of order $M+1$ with all diagonal elements being the constant $a$.

In the generalization to the multidomain situation, we assume that the computational domain is divided into $s$ subdomains. Under the $\beta$ formulation, the matrix eigenvalue equation without imposing boundary conditions across the subdomain boundaries can be written as

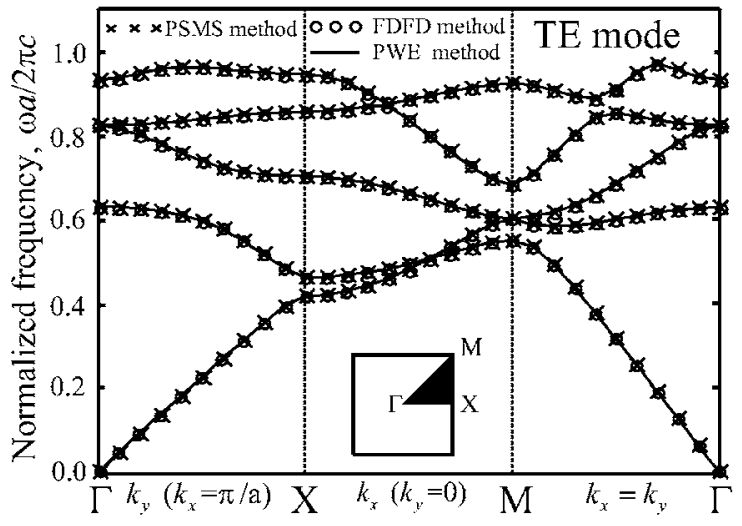

(a)

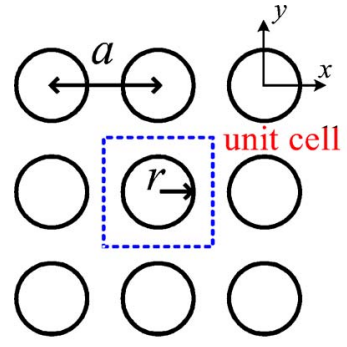

(a)

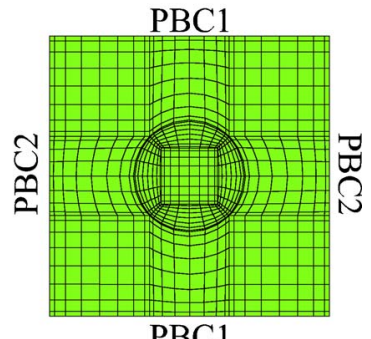

PBC1

(b)
FIG. 3. (Color online) (a) Cross section of a 2D square-lattice PC and its unit cell specified by the dashed lines. (b) Mesh and domain division profile for the unit cell.

$$
\begin{gathered}
{\left[\begin{array}{cccc}
{[P]_{1}} & \mathbf{0} & \cdots & \mathbf{0} \\
\mathbf{0} & {[P]_{2}} & \cdots & \mathbf{0} \\
\vdots & \vdots & \ddots & \vdots \\
\mathbf{0} & \mathbf{0} & \cdots & {[P]_{s}}
\end{array}\right]_{[s(M+1)(N+1)] \times[s(M+1)(N+1)]}=-k_{0}^{2}\left[\begin{array}{c}
\{\phi\}_{1} \\
\vdots \\
\{ \\
\{\phi\}_{s}
\end{array}\right]_{[s(M+1)(N+1)] \times 1}} \\
\times\left[\begin{array}{c}
\{\phi\}_{1} \\
\vdots\}_{2} \\
\vdots \\
\{\phi\}_{s}
\end{array}\right]_{[s(M+1)(N+1)] \times 1}
\end{gathered}
$$

where the subscript of $[P]_{i}(i=1,2, \ldots, s)$ represents the numbering of each subdomain. Then we can connect the adjacent subdomains by imposing the boundary conditions and possible PBCs on those matrix elements in Eq. (20) that correspond to the boundary nodes of the subdomain. In our numerical scheme, we have intentionally applied different types of boundary conditions (Dirichlet or Neumann type) to the adjacent subdomains to guarantee numerical stability. In the Appendix, we show how Eq. (20) will be modified when the boundary conditions are imposed. Finally, the field distributions (eigenvectors) and the eigenfrequencies (eigenvalues) can be obtained by using the SIPM.

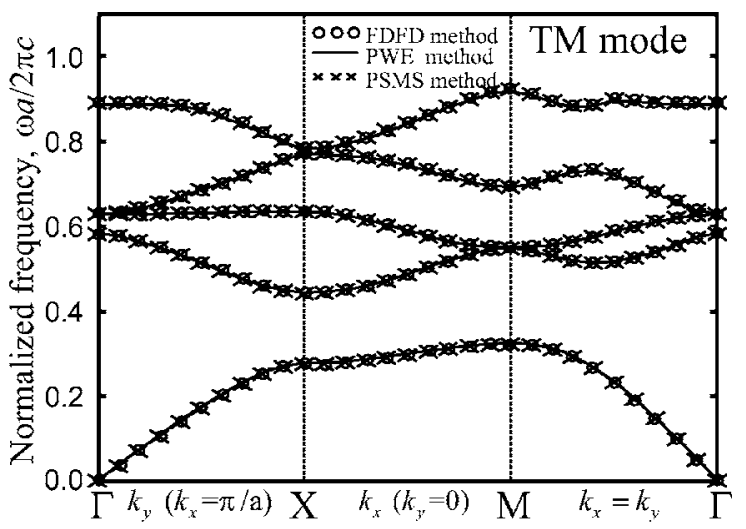

(b)

FIG. 4. 2D PC band diagrams involving square-arranged alumina rods with $r / a=0.2$ and $n=(8.9)^{1 / 2}$ in the air. (a) TE and (b) TM mode. 
TABLE I. Normalized frequencies of TE first and second bands at the $M$ point for the square-lattice PC obtained with different polynomial degrees.

\begin{tabular}{lccc}
\hline \hline & & \multicolumn{2}{c}{ Normalized frequency $(\omega a / 2 \pi c)$} \\
\cline { 3 - 4 }$N$ & Grid points & TE first mode & TE second mode \\
\hline 4 & 325 & 0.548961563774 & 0.603564998825 \\
5 & 468 & 0.548847662048 & 0.602451830831 \\
6 & 637 & 0.548861905144 & 0.601878879371 \\
8 & 1053 & 0.548843381514 & 0.601903085785 \\
12 & 2197 & 0.548843155002 & 0.601898903235 \\
14 & 2925 & 0.548843160037 & 0.601898894833 \\
18 & 4693 & 0.548843160882 & 0.601898894955 \\
20 & 5733 & 0.548843160880 & 0.601898894965 \\
\hline \hline
\end{tabular}

\section{NUMERICAL EXAMPLES}

We present in this section five numerical examples to demonstrate the inherent accuracy and excellent numerical convergence behavior of the PSMS in the analysis of 2D PCs. Comparison will be made with the FDFD method [11] and the PWE method in the first four examples. The first two, the square-lattice and the triangular-lattice PCs, are those discussed in [11]. The third one is a PC having a large air hole in the unit cell. The fourth one relates to a PC composed of large dielectric pixels designed to possess large absolute band gaps [24]. The last example discusses the analysis of a mini band gap.

\section{A. PC with square lattice}

We first investigate the square-arranged 2D PC, with its cross section in the $x-y$ plane as shown in Fig. 3(a), formed by parallel alumina rods with refractive index $n=8.9^{1 / 2}$ and radius $r=0.2 a$ in the air, where $a$ is the lattice distance. The unit cell is specified by the dashed lines. The unit cell is divided into 13 subdomains, as shown in Fig. 3(b), with the four corner subdomains and the central subdomain being in a square shape and the other eight subdomains not in any rectangular shape. The mesh pattern of each subdomain shown is for $M=N=8$. When applying the boundary conditions Eqs. (3) and (6) at the boundaries of the unit cell, the following PBCs need to be carefully taken into account:

$$
\begin{aligned}
& \psi(x, y+a)=e^{-j k_{y} a} \psi(x, y) \quad(\mathrm{PBC} 1), \\
& \psi(x+a, y)=e^{-j k_{x} a} \psi(x, y) \quad(\mathrm{PBC} 2),
\end{aligned}
$$

where $k_{x}$ and $k_{y}$ are the wave numbers in the $x$ and $y$ directions, respectively, and the boundaries at which the PBC1 or the PBC2 applies are indicated in Fig. 3(b). The calculated band diagrams of the TE and TM modes are plotted in Figs. 4(a) and 4(b), respectively. Each point along the boundary of the first Brillouin zone shown as the inset in Fig. 4(a) provides $k_{x}$ and $k_{y}$ in Eq. (21). The crosses are the results obtained using our PSMS based on Chebyshev polynomials of degree $12(M=N=12)$. (In fact, using just $M=N=4$, the obtained results would be indistinguishable when plotted, as can be seen in the later discussion.) The circles are the results of the compact FDFD algorithm with the index average scheme and using 40 grid points in each lattice distance [11]

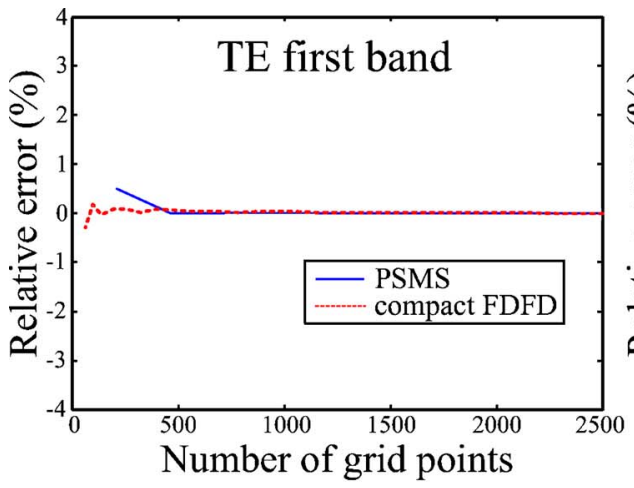

(a)

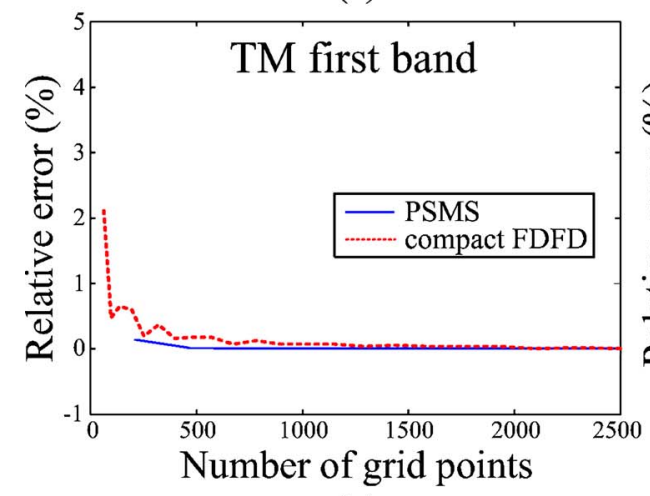

(c)

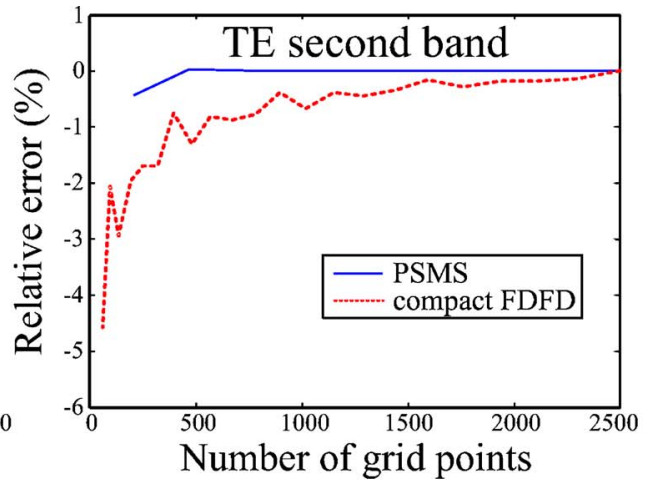

(b)

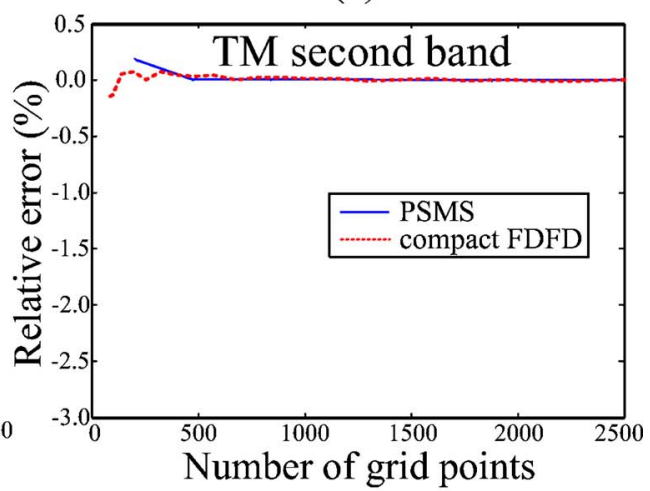

(d)
FIG. 5. (Color online) Convergence properties of the proposed PSMS method for the 2D squarelattice PC compared with the compact FDFD method [11]. (a) TE first band; (b) TE second band; (c) TM first band; (d) TM second band. 


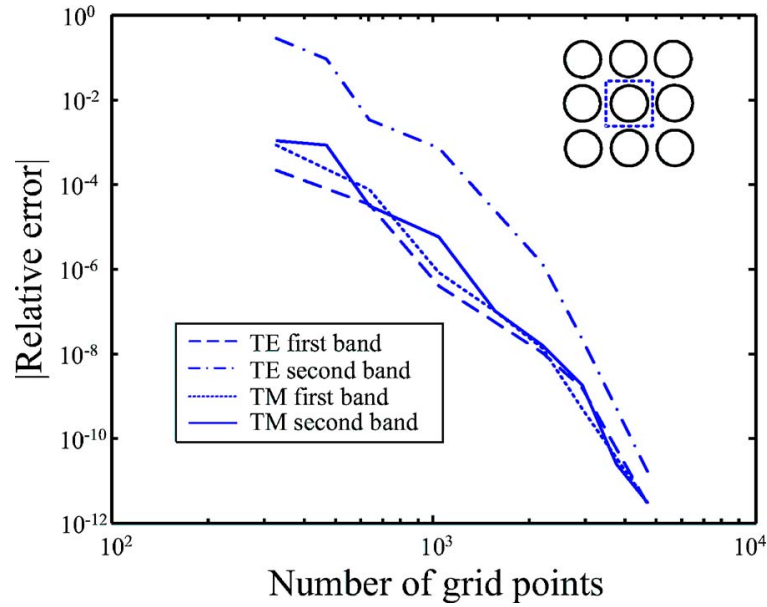

FIG. 6. (Color online) Convergence properties of the proposed PSMS method for the four bands of Fig. 5 plotted with the absolute value of the relative error in $\log -\log$ scale.

and the solid lines are obtained using the MIT photonicbands package [14] based on the PWE method with 128 $\times 128$ resolution. It is seen that the three results agree with one another quite well for both polarizations.

To examine the numerical convergence behavior, we first list in Table I the calculated normalized eigenfrequencies $(\omega a / 2 \pi c)$ of the TE first and second bands at the $M$ point in the first Brillouin zone, i.e., $k_{x}=\pi / a$ and $k_{y}=\pi / a$, for different degrees $(M=N)$ of the Chebyshev polynomials and the corresponding numbers of grid points within the unit cell. It is seen that results with three to four digits of accuracy can easily be obtained using hundreds of grid points and convergence up to $10^{-10}$, though not necessary for practical purposes, can be achieved by using high degrees. In [11], the accuracy of the compact FDFD algorithm with the index average scheme was examined for the analysis of the first and second bands of both the TE and TM modes and it was found that the speeds of numerical convergence were not uniformly similar among these four bands. We show in Figs. 5(a)-5(d) the relative errors of these four band frequencies at the $M$ point versus the number of grid points within the unit cell calculated using the PSMS and the compact FDFD method [11], respectively. The relative error refers to the difference between a calculated value and a reference value obtained by taking 2500 grid points. The convergence of the compact FDFD method is relatively slow for the TE second band in Fig. 5(b). However, it is seen that the convergence behavior of the PSMS is uniformly excellent for all four bands. This should be attributed to the inherent high-order scheme of the pseudospectral method as well as the rigorous satisfaction of the continuity conditions across the curved dielectric interfaces in our proposed formulation. To more clearly demonstrate the excellent convergence behavior of the PSMS, we show in Fig. 6 in log-log scale the absolute values of relative errors of the four band frequencies in Fig. 5 obtained by the PSMS versus the number of grid points within the unit cell, referring to the same reference values as mentioned above.

Some discussion regarding numerical convergence of the PWE method and the FDFD method and the role of the index average (IA) scheme is given below. In the IA scheme, the permittivity at a grid point $(i, j)$ is defined as $\epsilon(i, j)=f \epsilon_{1}$ $+(1-f) \epsilon_{2}$, where $\epsilon_{1}$ and $\epsilon_{2}$ are the permittivities of media 1 and 2, respectively, in the elementary grid mesh with the grid point located at its center, and $f$ is the filling fraction of medium 1 in the mesh. Another version of the average scheme is the inverse IA scheme, which involves a weighted average of the inverses of the permittivities, i.e., $1 / \epsilon(i, j)$ $=f / \epsilon_{1}+(1-f) / \epsilon_{2}$. Figures $7(\mathrm{a})$ and $7(\mathrm{~b})$ shows the relative errors of the TE first and second band frequencies, respectively, at the $M$ point versus the number of grid points within the unit cell obtained using the MPB package, the compact FDFD method with the IA scheme, and the compact FDFD method with the inverse IA scheme. The relative error here refers to the difference between a calculated value and a reference value obtained by taking 4096 grid points. Figures 7(a) and 7(b) are related to Figs. 5(a) and 5(b), respectively. The MPB package is believed to employ the inverse IA scheme [14]. As one has already observed in Figs. 5(a) and 5(b), the FDFD method with the IA scheme performs very well for the TE first band but shows slow convergence for the TE second band. It is interesting to see that when using the FDFD method with the inverse IA scheme, the situation reverses and the convergence in the TE first band is now slow. The MPB package results appear to have quite similar convergence performance in Figs. 7(a) and 7(b) but show oscillatory behavior. Therefore, the superior convergence characteristics of the PSMS should be appreciated.

Regarding computational time, we use the case of Fig. 4(a) as an example, where five bands were calculated with 30

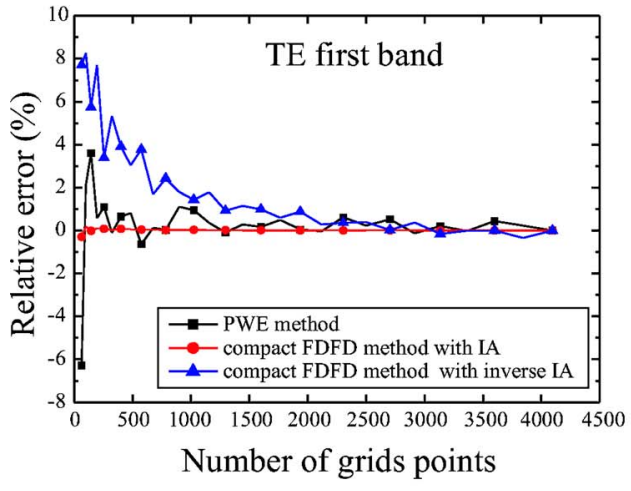

(a)

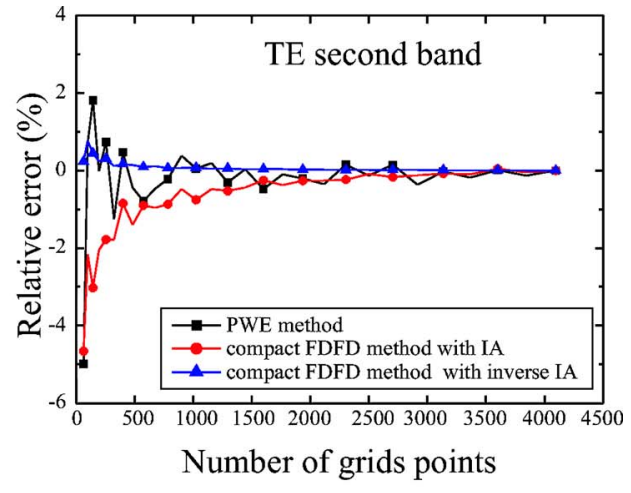

(b)
FIG. 7. (Color online) Comparison of convergence properties among the PWE method, the compact FDFD method with the IA scheme, and the comapct FDFD method with the inverse IA scheme for the 2D square-lattice PC. TE (a) first and (b) second band. 
(a)

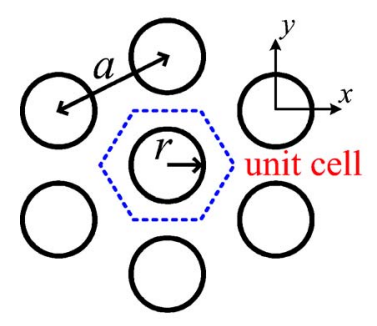

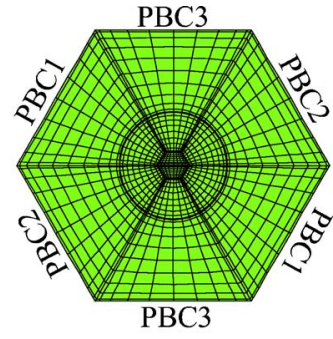

(b)
FIG. 8. (Color online) (a) Cross section of a 2D triangularlattice PC and its unit cell specified by the dashed lines. (b) Mesh and domain division profile for the unit cell.

data points for each band, i.e., 150 eigenfrequencies were determined. It took $83 \mathrm{~s}$ for the degree-4 $(M=N=4)$ scheme and $147 \mathrm{~s}$ for the degree- 6 scheme on Pentium IV $3.0 \mathrm{GHz}$ personal computers. Such computational cost is comparable to that of a typical PWE or FDFD calculation. But it can be observed from Fig. 5 that the relative errors are already within $0.5 \%$ for the degree- 4 results and that the degree- 6 results are almost indistinguishable from the final ones.

\section{B. PC with triangular lattice}

The second example is a 2D PC with triangular lattice, with its cross section in the $x-y$ plane as shown in Fig. 8(a), composed of dielectric cylinders with refractive index $n$ $=(11.4)^{1 / 2}$ and radius $r=0.2 a$ in the air. The unit cell is indicated in Fig. 8(a) and is shown in Fig. 8(b) with 14 subdomains in the computational domain division. Here, the mesh pattern of each subdomain shown is for $M=N=10$. The following PBCs need to be applied to the boundaries of the unit cell

$$
\begin{aligned}
& \psi\left(x+\frac{\sqrt{3} a}{2}, y-\frac{a}{2}\right)=e^{-j\left(k_{x} \sqrt{3} a / 2-k_{y} a / 2\right)} \psi(x, y) \quad(\mathrm{PBC} 1), \\
& \psi\left(x+\frac{\sqrt{3} a}{2}, y+\frac{a}{2}\right)=e^{-j\left(k_{x} \sqrt{3} a / 2+k_{y} a / 2\right)} \psi(x, y) \quad(\mathrm{PBC} 2),
\end{aligned}
$$

$$
\psi(x, y+a)=e^{-j k_{y} a} \psi(x, y) \quad(\mathrm{PBC} 3)
$$

The sides of the unit-cell boundaries at which the PBC1, the $\mathrm{PBC} 2$, or the $\mathrm{PBC} 3$ applies are indicated in Fig. 8(b). Figures 9(a) and 9(b) show the calculated band diagrams for the TE and TM modes, respectively. Each point along the boundary of the first Brillouin zone shown as the inset in Fig. 9(a) provides $k_{x}$ and $k_{y}$ in (22). The crosses are the results obtained using our PSMS based on Chebyshev polynomials of degree $12(M=N=12)$. The circles are the results of the compact FDFD algorithm with the index average scheme [11] and the solid lines are obtained by the MPB package based on the PWE method [14]. Again, fairly good agreement is observed among these three results for both polarizations. To examine the numerical convergence characteristics, we list in Table II the calculated normalized eigenfrequencies $(\omega a / 2 \pi c)$ of the TE first and second bands at the $K$ point in the first Brillouin zone shown as the inset in Fig. 9(a), i.e., $k_{x}=2 \pi / \sqrt{3} a$ and $k_{y}=2 \pi / 3 a$, for different degrees $(M=N)$ of the Chebyshev polynomials and the corresponding numbers of grid points within the unit cell. As in Table I, we observe that results with three to four digits of accuracy can easily be obtained using hundreds of grid points and higher accuracy can be achieved by using high degrees. As in Figs. 5(a)-5(d), we plot in Figs. 10(a)-10(d) the relative errors of the eigenfrequencies at the $K$ point versus the number of grid points within the unit cell for the first and second bands of the TE and TM modes calculated using the PSMS and the compact FDFD method [11]. Again, the convergence of the compact FDFD method is seen to be relatively slow for the TE second band in Fig. 10(b) and the convergence behavior of the PSMS is uniformly excellent for all four bands. As in Fig. 6, the absolute values of relative errors versus the number of grid points are presented in log-log scale in Fig. 11 for the four bands of Fig. 10.

\section{PC with large air holes}

We consider a square-lattice PC having a large air hole in the unit cell. The cross section is shown in Fig. 12(a), where $n_{b}=1$, the radius of the air hole is $r=0.45 a$, and the background is a material with $n_{a}=(12.96)^{1 / 2}$. Since the distance between the edges of adjacent air holes is small, the analysis using FD methods would involve much finer mesh grids and enormous numbers of grids are required when the uniform grid scheme is employed. Using our PSMS, this difficulty is less severe. We again divide the area of the unit cell into 13

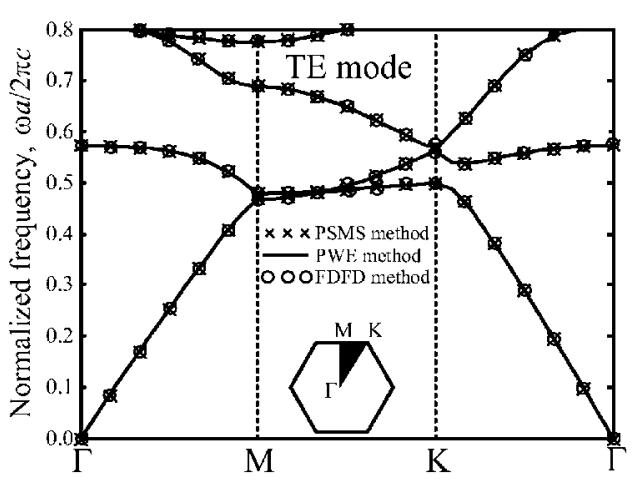

(a)

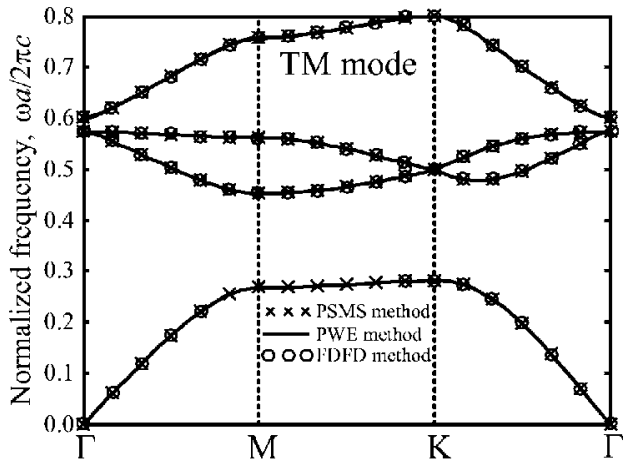

(b)
FIG. 9. Comparison of band diagram calculations obtained using different methods for the 2D $\mathrm{PC}$ formed by triangular-arranged dielectric cylinders with $r / a=0.2$ and $n=(11.4)^{1 / 2}$ in the air. (a) TE and (b) TM mode. 
TABLE II. Normalized frequencies of TE first and second bands at the $K$ point for the triangular-lattice $\mathrm{PC}$ obtained with different polynomial degrees.

\begin{tabular}{lccc}
\cline { 3 - 4 }$N$ & Grid points & TE first mode & TE second mode \\
\hline 4 & 350 & 0.499283589912 & 0.564109638904 \\
5 & 504 & 0.499205727643 & 0.564270988892 \\
6 & 686 & 0.499105436444 & 0.564174744801 \\
8 & 1134 & 0.499105213003 & 0.564148913039 \\
12 & 2366 & 0.499105288316 & 0.564150042905 \\
14 & 3150 & 0.499105289745 & 0.564150079536 \\
18 & 5054 & 0.499105289699 & 0.564150087486 \\
20 & 6174 & 0.499105289694 & 0.564150087334 \\
\hline \hline
\end{tabular}

subdomains, as shown in Fig. 12(b) with the mesh pattern of each subdomain corresponding to $M=N=10$. Table III lists the calculated normalized eigenfrequencies $(\omega a / 2 \pi c)$ of the TE first and second bands at the $M$ point in the first Brillouin zone for different degrees $(M=N)$ of the Chebyshev polynomials and the corresponding numbers of grid points within the unit cell. The PSMS can still provide rapid convergence using only hundreds of grid points and accuracy up to $10^{-7}$ is observed for $M=N=20$.

\section{PC with large dielectric pixels}

Recently, Shen et al. [24] reported a 2D PC structure formed by large dielectric pixels that possesses a large absolute band gap in the frequency region $0 \leqslant \omega a / 2 \pi c \leqslant 1$. The structure was found by searching over many candidates based on $10 \times 10$ pixels in the unit cell. The cross section of the structure is shown in Fig. 13(a) with the unit cell indicated by the dashed lines. The gray pixels were filled with GaAs $\left[n=(11.4)^{1 / 2}\right]$ and others are air. Each pixel is treated as a subdomain in our PSMS analysis and thus there are 100 subdomains in the unit cell, as shown in Fig. 13(b). The mesh pattern of each subdomain shown is for $M=N=4$. Shen et al. introduced a fast PWE method for analyzing the TE modes of such special PC structures for which $\epsilon E_{x}$ is always continuous in $x$ and $E_{x}$ is continuous in $y$. They obtained eigenfrequencies for the TE first and eleventh bands at the $M$ point in the first Brillouin zone of $0.3893(2 \pi c / a)$ and $1.299(2 \pi c / a)$, respectively. The frequency span of the large absolute band gap they found was $0.093(2 \pi c / a)$ with a midfrequency at $0.71(2 \pi c / a)$. In our analysis using the PSMS, no special modification of the scheme is needed. Our results for the same normalized eigenfrequencies of the same TE first and eleventh bands are listed in Table IV for different degrees $(M=N)$ of the Chebyshev polynomials and the corresponding numbers of grid points within the unit cell. It can be seen that by simply using $M=N=3$, the differences from the high-order results $(M=N=13)$ are only $0.6 \%$ and $1.36 \%$ for the first and eleventh bands, respectively. Our results up to four-digit accuracy are $0.3889(2 \pi c / a)$ and $1.296(2 \pi c / a)$, which are $0.1 \%$ to $0.2 \%$ different from the above-mentioned values in [24]. The band diagrams for the TE and TM modes calculated using the PSMS are plotted in Figs. 14(a) and 14(b), respectively, with the absolute band gap indicated by

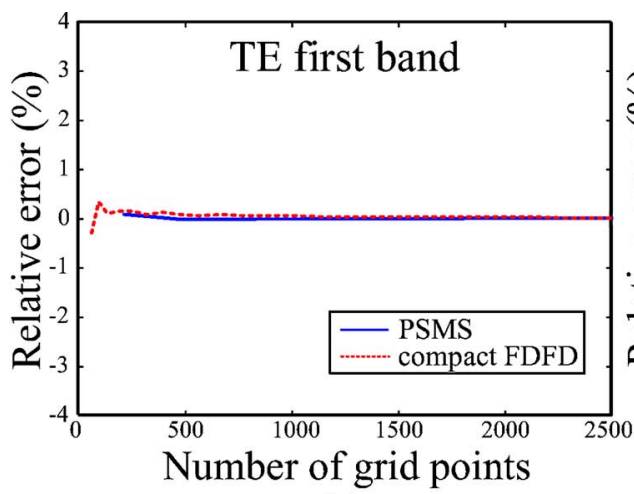

(a)

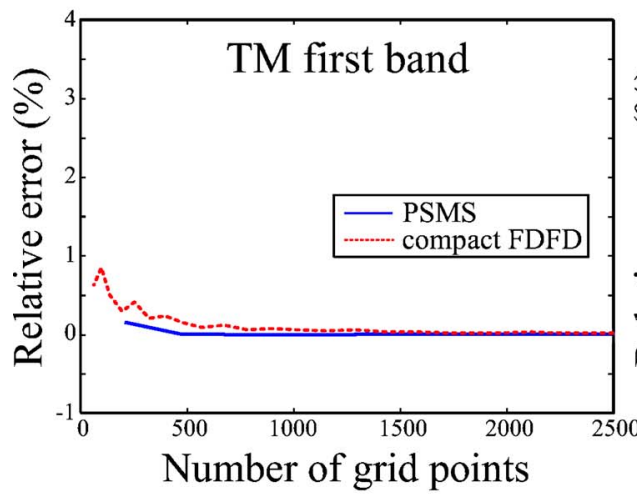

(c)

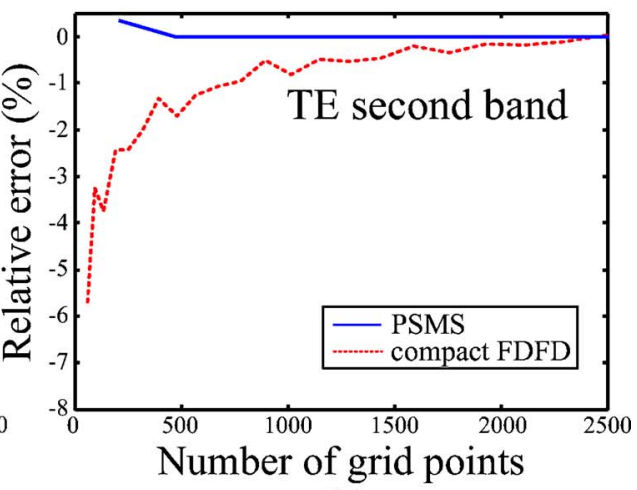

(b)

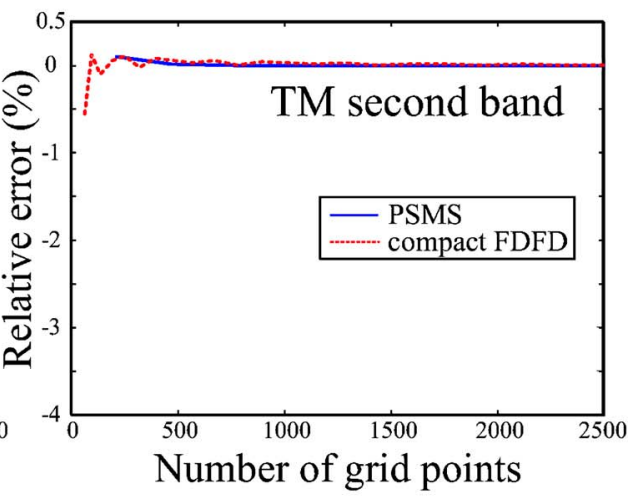

(d)
FIG. 10. (Color online) Convergence properties of the proposed PSMS method for the 2D triangular-lattice PC compared with the compact FDFD method [11]. (a) TE first band; (b) TE second band; (c) TM first band; (d) TM second band. 


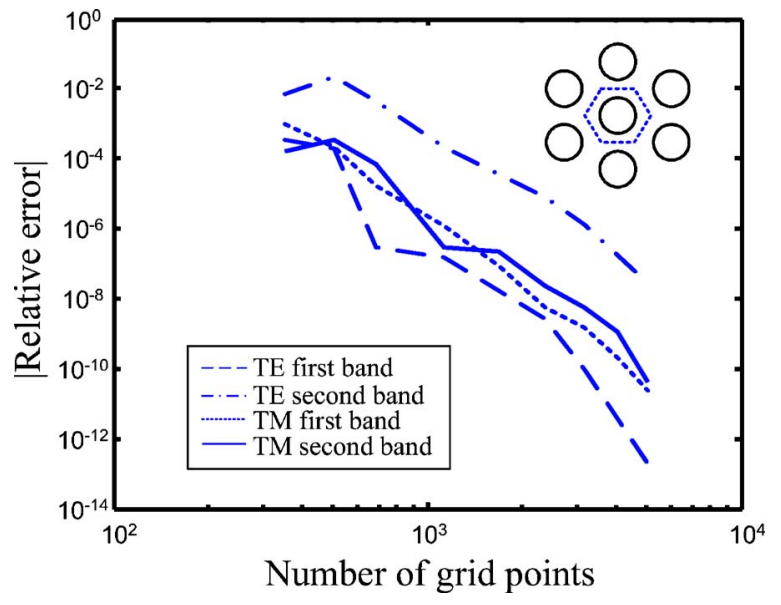

FIG. 11. (Color online) Convergence properties of the proposed PSMS method for the four bands of Fig. 10 plotted with the absolute value of the relative error in $\log$-log scale.

the gray area. The width of the gap is $0.0927(2 \pi c / a)$ in our analysis, compared to $0.093(2 \pi c / a)$ in [24].

\section{E. Analysis of a mini band gap}

Due to the high accuracy of the PSMS analysis, we are able to resolve very small band gaps. We finally show an example of the analysis of a mini band gap. Consider the mini gap between the TM fourth and fifth bands at the $X$ point in Fig. 4(b) (the square-arranged alumina rods case with $r / a=0.2$ ), where the normalized frequency gap width is $\Delta \omega a / 2 \pi c=0.014946$. We vary the value of $r / a$ and study the corresponding gap width change for this mini gap. Figure 15(a) shows the variations of the normalized frequencies of these two bands at the $X$ point versus $r / a$. The line of circles means the band has mode pattern $A$ as shown in Fig. 15(b) and the line of triangles means the band has mode pattern $B$ as shown in Fig. 15(c). The mode patterns $A$ and $B$ are plotted as constant- $E_{z}$ contours normalized to the (absolute) peak value with the thin solid lines representing the zero-value contours and the dashed lines corresponding to negative $E_{z}$ values of $-0.2,-0.5,-0.7,-0.8$, and -0.9 , progressively. In mode pattern $A$, the three thick solid lines correspond to positive $E_{z}$ values of $0.2,0.5$, and 0.6 , progressively, and in

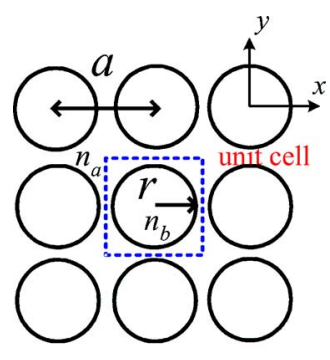

(a)

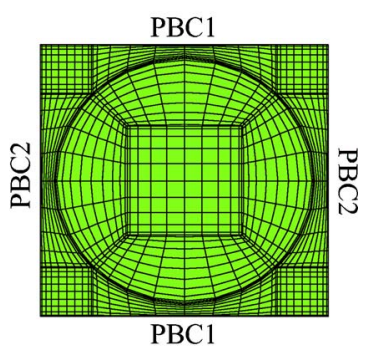

(b)
FIG. 12. (Color online) (a) Cross section of a 2D square-lattice PC with $r / a=0.45, n_{b}=1$, and $n_{a}=(12.96)^{1 / 2}$. (b) Mesh and domain division profile for the unit cell.
TABLE III. Normalized frequencies of TE first and second bands at the $M$ point for the large-air-hole PC obtained with different polynomial degrees.

\begin{tabular}{lccc}
\hline \hline & & \multicolumn{2}{c}{ Normalized frequency $(\omega a / 2 \pi c)$} \\
\cline { 3 - 4 }$N$ & Grid points & TM first mode & TM second mode \\
\hline 3 & 208 & 0.229982620857 & 0.288057199628 \\
4 & 325 & 0.220672221696 & 0.290216083144 \\
6 & 637 & 0.219621158554 & 0.291714207681 \\
8 & 1053 & 0.220451988363 & 0.291061620411 \\
12 & 2197 & 0.220323306279 & 0.291156559374 \\
14 & 2925 & 0.220318821829 & 0.291157377619 \\
18 & 4693 & 0.220319456275 & 0.291157404741 \\
20 & 5733 & 0.220319475518 & 0.291157420884 \\
\hline \hline
\end{tabular}

mode pattern $B$, the thick solid lines correspond to positive $E_{z}$ values of $0.2,0.5,0.7,0.8$, and 0.9 , progressively. It is interesting to observe that the mode patterns switch roles somewhere between $r / a=0.18665$ and 0.18670 , i.e., the higher-frequency band changes its pattern across this region and so does the lower-frequency band. The inset in Fig. 15(a) gives an enlarged plot of this crossover region. The normalized frequency gap width $\Delta \omega a / 2 \pi c$ is plotted in Fig. 16 as a function of $\delta=r / a-0.1866577$. In practical applications, the value of $r / a$ with seven digits after the decimal point might not be meanful, but here we just want to demonstrate the high-accuracy capability of our analysis. We thus show the normalized gap width down to the order of $10^{-7}$.

We observe one characteristic of the mode patterns that in the range of Fig. 16, although mode pattern $A$ maintains its shape as that shown in Fig. 15(b), mode pattern $B$ appears to lose its left-right symmetry near $\delta=0$. Figure 17 shows mode patterns $B$ at six points, $P_{1}$ to $P_{6}$, denoted in Fig. 16. The contour levels are the same as those in Fig. 15(c). The loss of left-right symmetry is clearly seen at points $P_{2}, P_{3}, P_{4}$, and $P_{5}$. For the four data points $P_{3}, P_{4}$, and the two nearest to them in Fig. 16, we use PSMS of degree $M=N=14$ to obtain converged results. For other points in the same figure, degree 10 is enough. In fact, we have computed the gap for $\delta=0$ and

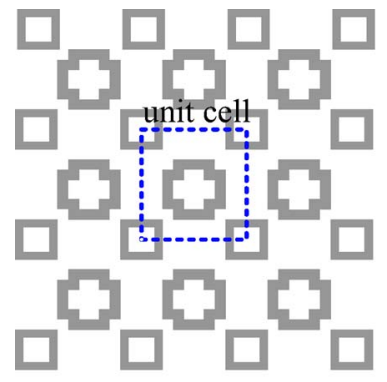

(a)

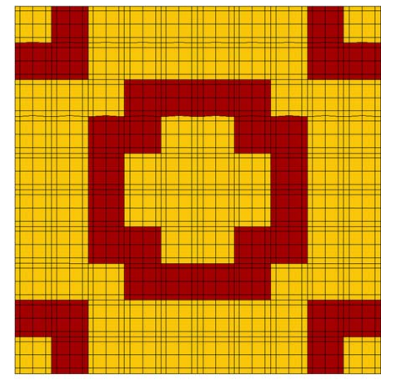

(b)
FIG. 13. (Color online) (a) Cross section of a 2D PC formed by large dielectric pixels reported in [24] and its unit cell specified by the dashed lines. (b) Mesh and domain division profile for the unit cell. 
TABLE IV. Normalized frequencies of TE first and eleventh bands at the $M$ point for the PC of Fig. 13 obtained with different polynomial degrees.

\begin{tabular}{lccc}
\hline \hline & & \multicolumn{2}{c}{ Normalized frequency $(\omega a / 2 \pi c)$} \\
\cline { 3 - 4 }$N$ & Grid points & First mode & Eleventh mode \\
\hline 3 & 1600 & 0.386564781760 & 1.278455023882 \\
6 & 4900 & 0.388675486464 & 1.294990564406 \\
8 & 8100 & 0.388818441981 & 1.295716582941 \\
10 & 12100 & 0.388868307141 & 1.295974347129 \\
12 & 16900 & 0.388890312409 & 1.296088577065 \\
13 & 19600 & 0.388901559911 & 1.296147017684 \\
\hline \hline
\end{tabular}

the obtained $\Delta \omega a / 2 \pi c$ is on the order of $9 \times 10^{-9}$ based on a degree-20 calculation.

\section{CONCLUSION}

We have proposed and formulated an analysis method for obtaining the band diagrams of 2D PCs based on a multidomain pseudospectral method. The method is an eigenmode solver and is named the pseudospectral mode solver. We have utilized the multidomain Chebyshev collocation method by which Chebyshev-Lagrange interpolating polynomials are employed in the approximation of spatial derivatives at collocation points and then the Helmholtz equation is converted into a matrix eigenvalue equation. The eigenfrequencies of the band structures were solved by the shift inverse power method. Through the multidomain scheme and a curvilinear coordinate mapping technique, the computational domain was divided into a suitable number of subdomains having curved shapes to fit the general curved interfaces of the permittivity profile. Alternative Dirichlet and Neumann type boundary conditions between adjacent subdomains have been derived and applied to assure the numerical stability and accuracy. Four numerical examples, including squarelattice and triangular-lattice PCs, a PC having a large air hole in the unit cell, and a PC composed of large dielectric pixels, have been presented to demonstrate the uniformly excellent numerical convergence behavior and accuracy of the proposed method for both TE and TM waves. Such superior performance is attributed to the inherent high-order scheme of the pseudospectral method as well as the rigorous satisfaction of the continuity conditions across the curved dielectric interfaces. The proposed method thus has no problem in treating structures involving large refractive-index contrast as demonstrated in the numerical examples. Finally, a mini band gap has been analyzed for demonstrating the calculation of a normalized frequency gap width as small as on the order of $10^{-7}$.

\section{ACKNOWLEDGMENTS}

This work was supported in part by the National Science Council of the Republic of China under Grant No. NSC942215-E-002-022, in part by the Ministry of Education of the Republic of China under an "Aim of Top University Plan" grant, and in part by the Ministry of Economic Affairs of the Republic of China under Grant No. 94-EC-17-A-08-S10006. The authors would like to acknowledge the National Center for High-Performance Computing in Hsinchu, Taiwan for providing useful computing resources.

\section{APPENDIX: IMPLEMENTATION OF BOUNDARY CONDITIONS BETWEEN ADJACENT SUBDOMAINS}

We demonstrate how the matrix equation (20) will be modified when Dirichlet type and Neumann type boundary conditions are imposed at the interface between adjacent subdomains. For simplicity, we use a problem with only two subdomains, labeled as 1 and 2, as an example. Each subdomain contains $(M+1)(N+1)$ grid points with $(M+1)(N+1)$ corresponding field unknowns. Equation (20) now becomes

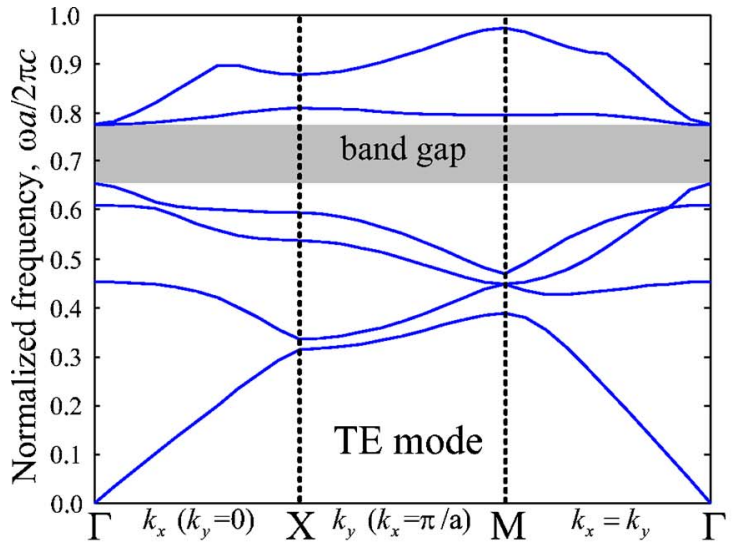

(a)

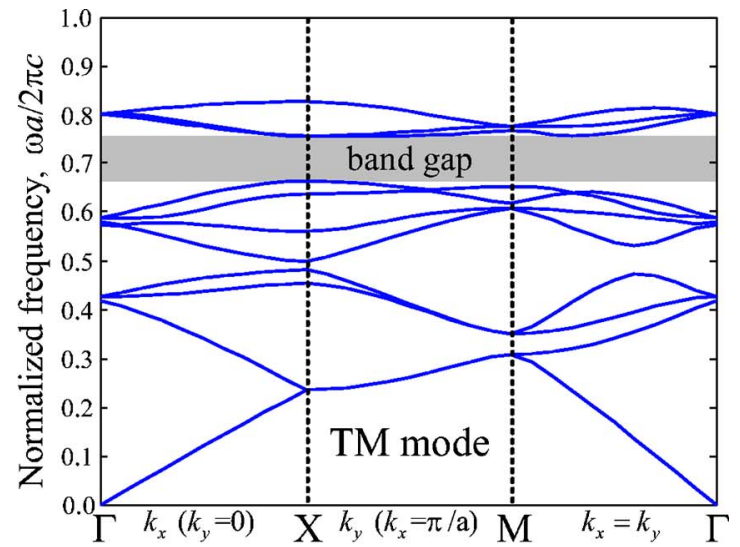

(b)

FIG. 14. (Color online) Band diagrams for the PC structure of Fig. 13. (a) TE and (b) TM mode. 


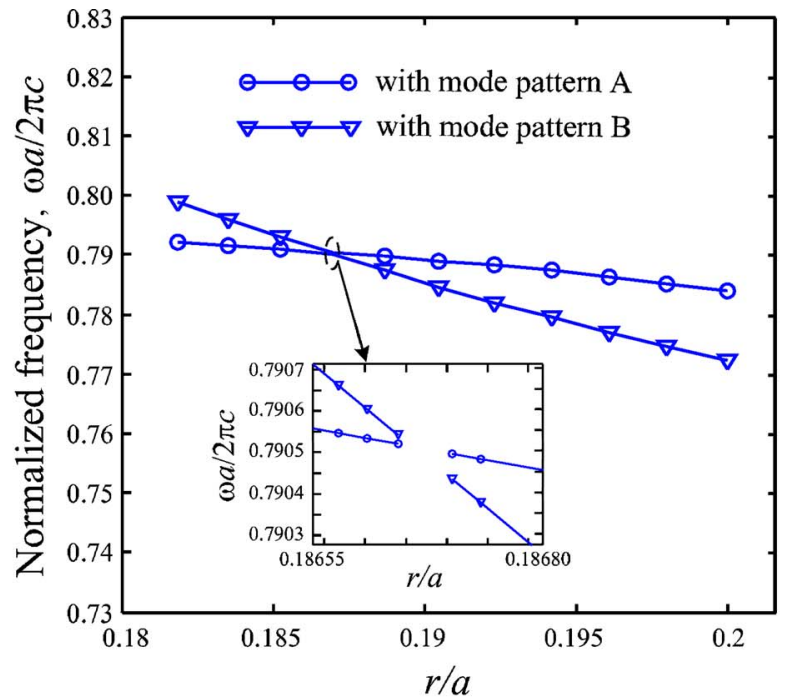

(a)

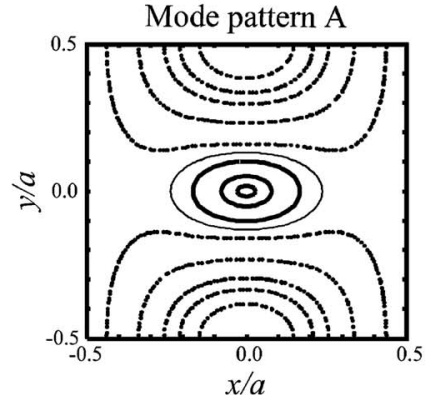

(b)

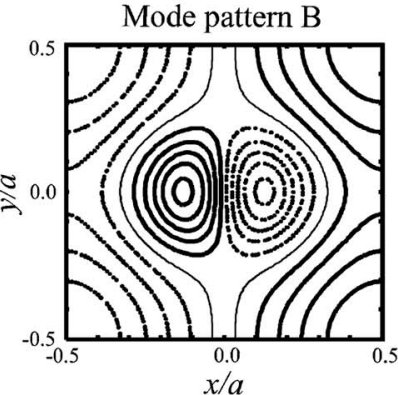

(c)
FIG. 15. (Color online) (a) Normalized frequencies of the TM fourth and fifth bands at the $X$ point in Fig. 4(b) for $r / a$ between 1.8 and 2.0. The inset gives an enlarged plot of the crossover region. The bands have mode patterns $A$ and $B$. (b) Constant $-E_{z}$ contours for mode pattern $A$. (c) Constant- $E_{z}$ contours for mode pattern $B$.

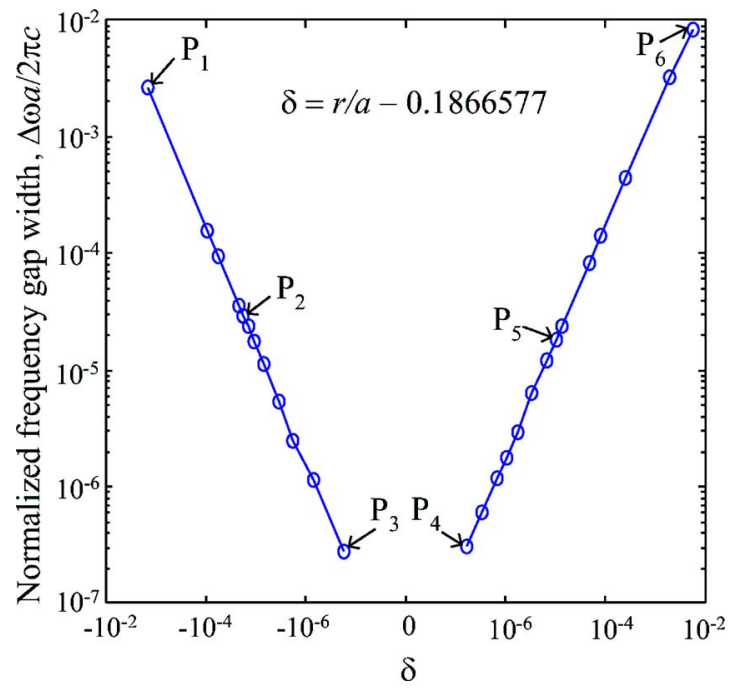

FIG. 16. (Color online) Normalized frequency gap width from the results of Fig. 15 near the crossover region plotted as a function of $\delta=r / a-0.1866577$.
Mode pattern B
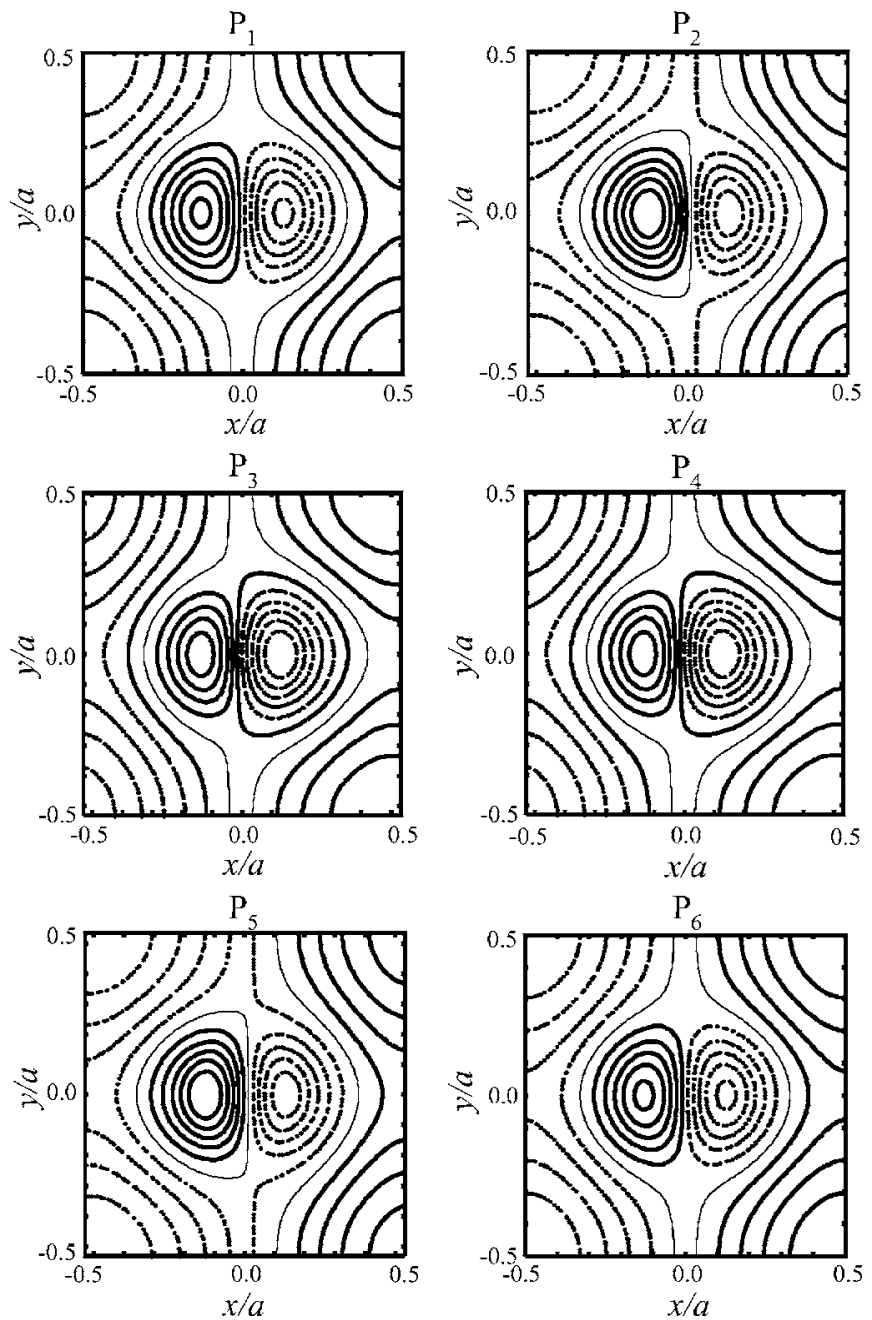

FIG. 17. Mode patterns $B$ at points $P_{1}$ to $P_{6}$ in Fig. 16 .

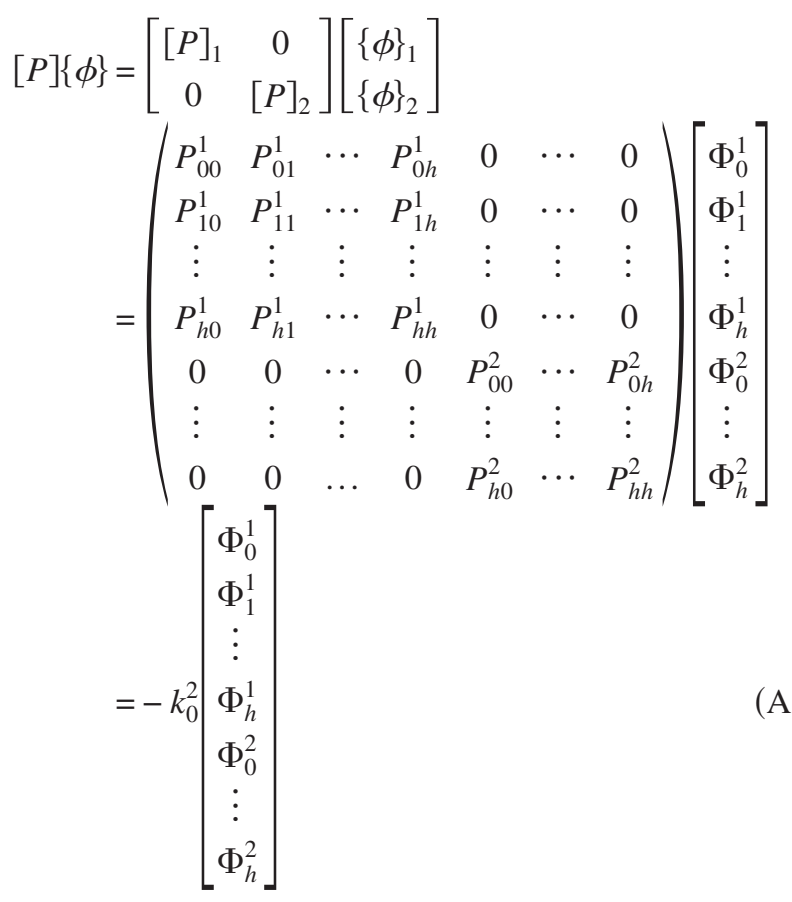


where $\Phi_{i}^{1}$ and $\Phi_{i}^{2}(i=0,1, \cdots, h)$ are the elements of $\{\phi\}_{1}$ and $\{\phi\}_{2}$, respectively, and $h=(M+1)(N+1)-1$. The spatial derivatives of $\{\phi\}$ can be obtained using a matrix operator as

$$
\begin{aligned}
\frac{\partial\{\phi\}}{\partial j} & =\left[D_{j}\right]\{\phi\}=\left[\begin{array}{cccc}
\overline{D_{j 00}} & 0 \\
0 & \bar{D}_{j 00}^{2}
\end{array}\right]\left[\begin{array}{c}
\{\phi\}_{1} \\
\{\phi\}_{2}
\end{array}\right] \\
& =\left(\begin{array}{ccccccc}
D_{j 00}^{1} & D_{j 01}^{1} & \cdots & D_{j 0 h}^{1} & 0 & \cdots & 0 \\
D_{j 10}^{1} & D_{j 11}^{1} & \cdots & D_{j 1 h}^{1} & 0 & \cdots & 0 \\
\vdots & \vdots & \vdots & \vdots & \vdots & \vdots & \vdots \\
D_{j h 0}^{1} & D_{j h 1}^{1} & \cdots & D_{j h h}^{1} & 0 & \cdots & 0 \\
0 & 0 & \cdots & 0 & D_{j 00}^{2} & \cdots & D_{j 0 h}^{2} \\
\vdots & \vdots & \vdots & \vdots & \vdots & \vdots & \vdots \\
0 & 0 & \cdots & 0 & D_{j h 0}^{2} & \cdots & D_{j h h}^{2}
\end{array}\right)\left[\begin{array}{c}
\Phi_{0}^{1} \\
\Phi_{1}^{1} \\
\vdots \\
\Phi_{h}^{1} \\
\Phi_{0}^{2} \\
\vdots \\
\Phi_{h}^{2}
\end{array}\right]
\end{aligned}
$$

where $j$ denotes $x$ or $y$, and $\overline{\bar{D}}_{j 00}^{1}$ or $\overline{\bar{D}}_{j 00}^{2}$ is $\overline{\bar{D}}_{j 00}$ of Eq. (19) defined in the corresponding subdomain. Consider a particular grid point at the interface, which corresponds to the $u$ th point of subdomain 1 and the $v$ th point of subdomain 2, and with unknown field values $\Phi_{u}^{1}$ and $\Phi_{v}^{2}$, respectively. Then, the discrete form of Eq. (3) is written as

$$
\Phi_{u}^{1}=\Phi_{v}^{2}
$$

for both TE and TM modes, and that of Eq. (6) as

$$
\begin{aligned}
& \left\{n_{x}\left[\begin{array}{llll}
D_{x u 0}^{1} & D_{x u 1}^{1} & \cdots & D_{x u h}^{1}
\end{array}\right]\right. \\
& \left.+n_{y}\left[\begin{array}{llll}
D_{y u 0}^{1} & D_{y u 1}^{1} & \cdots & D_{y u h}^{1}
\end{array}\right]\right\}\left[\begin{array}{c}
\Phi_{0}^{1} \\
\Phi_{1}^{1} \\
\vdots \\
\Phi_{h}^{1}
\end{array}\right] \\
& -\left(\frac{n_{a}}{n_{b}}\right)^{2}\left\{n_{x}\left[\begin{array}{llll}
D_{x v 0}^{2} & D_{x v 1}^{2} & \cdots & D_{x v h}^{2}
\end{array}\right]\right.
\end{aligned}
$$

$$
\left.+n_{y}\left[\begin{array}{llll}
D_{y v 0}^{2} & D_{y v 1}^{2} & \cdots & D_{y v h}^{2}
\end{array}\right]\right\}\left[\begin{array}{c}
\Phi_{0}^{2} \\
\Phi_{1}^{2} \\
\vdots \\
\Phi_{h}^{2}
\end{array}\right]=0
$$

(A4a)

for the TE mode and

$$
\begin{aligned}
& \left\{n_{x}\left[\begin{array}{llll}
D_{x u 0}^{1} & D_{x u 1}^{1} & \cdots & D_{x u h}^{1}
\end{array}\right]\right. \\
& \left.+n_{y}\left[\begin{array}{llll}
D_{y u 0}^{1} & D_{y u 1}^{1} & \cdots & D_{y u h}^{1}
\end{array}\right]\right\}\left[\begin{array}{c}
\Phi_{0}^{1} \\
\Phi_{1}^{1} \\
\vdots \\
\Phi_{h}^{1}
\end{array}\right] \\
& -\left\{n_{x}\left[\begin{array}{llll}
D_{x v 0}^{2} & D_{x v 1}^{2} & \cdots & D_{x v h}^{2}
\end{array}\right]\right. \\
& \left.+n_{y}\left[\begin{array}{llll}
D_{y v 0}^{2} & D_{y v 1}^{2} & \cdots & D_{y v h}^{2}
\end{array}\right]\right\}\left[\begin{array}{c}
\Phi_{0}^{2} \\
\Phi_{1}^{2} \\
\vdots \\
\Phi_{h}^{2}
\end{array}\right]=0
\end{aligned}
$$

for the TM mode. Rewriting the $(u+1)$ th and $(h+v+1)$ th rows of Eq. (A1) using Eqs. (A3) and (A4), Eq. (A1) is modified to be

$$
\left.\left.\left(\begin{array}{cccccccccc}
P_{00}^{1} & \cdots & P_{0 u}^{1} & \cdots & P_{0 h}^{1} & 0 & \cdots & 0 & \cdots & 0 \\
\vdots & \vdots & \vdots & \vdots & \vdots & \vdots & \vdots & \vdots & \vdots & \vdots \\
0 & \cdots & 1 & 0 & \cdots & 0 & \cdots & -1 & 0 & 0 \\
P_{(u+1) 0}^{1} & \cdots & P_{(u+1) z}^{1} & \cdots & P_{(u+1) h}^{1} & 0 & \cdots & 0 & \cdots & 0 \\
\vdots & \vdots & \vdots & \vdots & \vdots & \vdots & \vdots & \vdots & \vdots & \vdots \\
0 & \cdots & 0 & \cdots & 0 & P_{00}^{2} & \cdots & P_{0 v}^{2} & \cdots & P_{0 h}^{2} \\
\vdots & \vdots & \vdots & \vdots & \vdots & \vdots & \vdots & \vdots & \vdots & \vdots \\
\mathcal{B}_{u 0}^{1 J} & \cdots & \mathcal{B}_{u u}^{1 J} & \cdots & \mathcal{B}_{u h}^{1 J} & \mathcal{B}_{v 0}^{2 J} & \cdots & \mathcal{B}_{v v}^{2 J} & \cdots & \mathcal{B}_{v h}^{2 J} \\
0 & \cdots & 0 & \cdots & 0 & P_{(v+1) 0}^{2} & \cdots & P_{(v+1) v}^{2} & \cdots & P_{(v+1) h}^{2} \\
\vdots & \vdots & \vdots & \vdots & \vdots & \vdots & \vdots & \vdots & \vdots & \vdots \\
0 & 0 & \cdots & 0 & \cdots & P_{h 0}^{2} & \cdots & P_{h v}^{2} & \cdots & P_{h h}^{2}
\end{array}\right)\left[\begin{array}{c}
\Phi_{0}^{1} \\
\vdots \\
\Phi_{u}^{1} \\
\Phi_{u+1}^{1} \\
\vdots \\
\Phi_{0}^{2} \\
\vdots \\
\Phi_{v}^{2} \\
\Phi_{v+1}^{2} \\
\vdots \\
\Phi_{h}^{2}
\end{array}\right]=-k_{0}^{2}\right] \begin{array}{c}
\Phi_{0}^{1} \\
\vdots \\
\Phi_{u-1}^{1} \\
0 \\
\Phi_{u+1}^{1} \\
\vdots \\
\Phi_{1}^{1} \\
\vdots \\
\Phi_{v-1}^{2} \\
0 \\
\Phi_{v+1}^{2} \\
\vdots \\
\Phi_{h}^{2}
\end{array}\right]
$$


where $J$ denotes the TE or TM mode, with $\mathcal{B}_{u i}^{1 \mathrm{TE}}=n_{x} D_{x u i}^{1}$ $+n_{y} D_{y u i}^{1}, \quad \mathcal{B}_{v i}^{2 \mathrm{TE}}=-\left(n_{a} / n_{b}\right)^{2}\left(n_{x} D_{x v i}^{2}+n_{y} D_{y v i}^{2}\right), \quad \mathcal{B}_{u i}^{1 \mathrm{TM}}=n_{x} D_{x u i}^{1}$ $+n_{y} D_{y u i}^{1}$, and $\mathcal{B}_{v i}^{2 \mathrm{TM}}=-\left(n_{x} D_{x v i}^{2}+n_{y} D_{y v i}^{2}\right)$, where $i=0,1, \cdots, h$.
Note that, after imposing the two boundary conditions at one grid point, the dimension of the matrix eigenvalue equation is reduced by 2 .
[1] E. Yablonovitch, Phys. Rev. Lett. 58, 2059 (1987).

[2] S. John, Phys. Rev. Lett. 58, 2486 (1987).

[3] J. D. Joannopoulos, R. D. Meade, and J. N. Winn, Photonic Crystal: Molding the Flow of Light (Princeton University Press, Princeton, NJ, 1995).

[4] K. M. Ho, C. T. Chan, and C. M. Soukoulis, Phys. Rev. Lett. 65, 3152 (1990).

[5] R. D. Meade, K. D. Brommer, A. M. Rappe, and J. D. Joannopoulous, Appl. Phys. Lett. 61, 495 (1992).

[6] M. Plihal and A. A. Maradudin, Phys. Rev. B 44, 8565 (1991).

[7] C. T. Chan, Q. L. Yu, and K. M. Ho, Phys. Rev. B 51, 16635 (1995).

[8] M. Qiu and S. He, J. Appl. Phys. 87, 8268 (2000).

[9] H. Y. D. Yang, IEEE Trans. Microwave Theory Tech. 34, 2688 (1996).

[10] L. Shen, S. He, and A. Xiao, Comput. Phys. Commun. 143, 213 (2002).

[11] C. P. Yu and H. C. Chang, Opt. Express 12, 1397 (2004).

[12] K. S. Yee, IEEE Trans. Antennas Propag. AP-14, 302 (1966).

[13] A. Yefet and E. Turkel, Appl. Numer. Math. 33, 125 (2000).
[14] S. G. Johnson and J. D. Joannopoulos, Opt. Express 8, 173 (2001).

[15] C. Canuto, M. Y. Hussani, A. Quarteroni, and T. Zang, Spectral Methods in Fluid Dynamics (Springer-Verlag, New York, 1988).

[16] B. Yang, and J. S. Hesthaven, IEEE Trans. Antennas Propag. 47, 132 (1999).

[17] J. S. Hesthaven, P. G. Dinesen, and J. P. Lynov, J. Comput. Phys. 155, 287 (1999).

[18] G. Zhao and Q. H. Liu, IEEE Trans. Antennas Propag. 52, 742 (2004).

[19] Q. H. Liu, IEEE Antennas Wireless Propag. Lett. 1, 131 (2002).

[20] W. J. Gordon and C. A. Hall, Numer. Math. 21, 109 (1973).

[21] G. R. Hadley, J. Lightwave Technol. 20, 1219 (2002).

[22] Y. C. Chiang, Y. P. Chiou, and H. C. Chang, J. Lightwave Technol. 20, 1609 (2002).

[23] M. Koashiba and Y. Tsuji, J. Lightwave Technol. 18, 737 (2000).

[24] L. Shen, S. He, and S. Xiao, Phys. Rev. B 66, 165315 (2002). 